\title{
Selective Alkylation of Aromatic Five-Membered Heterocyclic Molecules
}

\author{
D. SAMSONU ${ }^{1 *}$, M. BRAHMAYYA ${ }^{1,2}$, U. VIPLAVA PRASAD $^{1}$ and G. RAVI KUMAR ${ }^{2}$ \\ ${ }^{1}$ Department of Organic Chemistry and Foods, Drugs, Water Analysis, \\ Andhra University, Visakhapatnam -530003, India \\ ${ }^{2}$ Department of Engineering Chemistry, Andhra University, Visakhapatnam, India \\ samsonchem@gmail.com,brahma.orgchem@gmail.com
}

Received 14 November 2016 / Revised 24 December 2016 / Accepted 29 December 2016

\begin{abstract}
A series of alkylated heterocycles were selectively prepared from the corresponding heterocyclic compounds with the phase transfer catalyst tetraoctylammoniumbromide (TOAB), as catalyst at $100{ }^{0} \mathrm{C}$ temperatures. This present protocol demonstrates a simple alkylation procedure and has an extensive range of applicability and was applied to the furan, thiophene and pyrrole providing as another method for the research of these drug intermediates.
\end{abstract}

Keywords: Catalysis, Heterocyclic molecules, Methylation, Selectivity, Methodology

\section{Introduction}

Alkylated heterocycles are very important organic intermediates for the chemical industries such as drugs and pharmaceuticals ${ }^{1,2}$. 2-Methyl aromatic heterocycles was used for several medicinal chemistry purposes like anti-inflammatory antiviral drugs ${ }^{3}$. Many of pharmaceutical industries academic labs demands highly pure 2-alkylated 5-membered aromatic heterocycles of furan, thiophene and pyrrole. Furthermore, as the substitution of 3-alkyl heterocycles have drawback to the excellence of the final products, so the detachable of 3-alkyl substitution compound is a typical by-product in their reaction from the substrate compound which is a needed process that have important of special, very significant and monotonous determinations in the additional step i.e, 3-alkyl contaminating 2-alkyl furan should be removed by a discriminating electrophilic substitution procedure, bromination monitored by fractional distillation. About to the conventional alkylation procedure, heterocyclic molecules such as furan, thiophene and pyrrole were alkylated by Lewis acids and some other catalysts ${ }^{4,5}$. The 3 -alkyl substitution is the trivial contaminate presence is the extent of $20 \%$ in the alkylation procedure by straight forward using soluble acids. The major disadvantage like corrosive, factor of unrecovered catalyst and needed an additional stoichiometric quantities are the most common to use conventional Lewis acid catalysts, inorganic and organic acids in the Friedel-crafts alkylation reactions ${ }^{6-10}$. These catalyst also 
influence the durable complexion with the aimed product work up to decay the developed intermediate complex in the reaction by hydrolysis practices. leftover substrates or product that permits special attention in the mentioned catalysis systems. Even these methods needed long process and expensive too. These drawbacks have motivated to progress an environmental catalytic process for the alkylation fine chemicals using a nontoxic, that produces no sewage or impurities and concurrently to attain the higher range of choosiness of the 2-isomer to encounter the customer's wish. In this way we found a technique for the simple phase transfer alkylation ${ }^{7-9}$ with alkylating agents in presence of TOAB. The reaction between furan with methyl bromide at $100{ }^{\circ} \mathrm{C}$ on a tetraoctylammoniumbromide leads to 2alkyl furan with $98 \%$ selectivity at $99 \%$ conversion (Table 1).

Table 1. Selective alkylation of heterocyclic molecules

\begin{tabular}{ccccccc}
\hline $\begin{array}{c}\text { S. } \\
\text { No. }\end{array}$ & Heteroaromatic & Catalyst & $\begin{array}{c}\text { Temp., } \\
{ }^{\circ} \mathrm{C}\end{array}$ & $\begin{array}{c}\text { Time, } \\
\mathrm{h}\end{array}$ & $\begin{array}{c}\text { Conversion, } \\
\%^{\mathrm{a}}\end{array}$ & $\begin{array}{c}\text { Selectivity 2-acetyl } \\
\text { heteroaromatic, } \\
\text { Yield }\end{array}$ \\
\hline 1 & Thiophene & TOAB & RT & 6 & 50 & 40 \\
2 & Furan & TOAB & RT & 6 & 57 & 50 \\
3 & Pyrrole & TOAB & RT & 6 & 55 & 49 \\
4 & Thiophene & TOAB & 35 & 4 & 67 & 60 \\
5 & Furan & TOAB & 35 & 4.5 & 63 & 59 \\
6 & Pyrrole & TOAB & 35 & 4 & 69 & 63 \\
7 & Thiophene & TOAB & 40 & 3 & 78 & 72 \\
8 & Furan & TOAB & 40 & 3.5 & 80 & 78 \\
9 & Pyrrole & TOAB & 40 & 3.5 & 82 & 76 \\
10 & Thiophene & TOAB & 100 & 2 & 99 & 97 \\
11 & Furan & TOAB & 100 & 2 & 99 & 98 \\
12 & Furan & TOAB & 100 & 2 & 98 & 96 \\
\hline
\end{tabular}

${ }^{a}$ Based on methyl bromide, Conversion of the substrate to desired product on gas chromatography monitoring

\section{Experimental}

Alkylation of just five-heterocyclic aromatic moieties with methyl bromide in the presence of TOAB is a significant green approach to achieve these selected fine chemicals.

\section{Methods of characterizations for the molecules}

The formation of alkylated products was checked by the examination of reaction mass samplings, collected in sequence of time intervals by gas chromatography. ${ }^{1} \mathrm{H}$ NMR (400 $\mathrm{MHz}$ ) spectra were recorded on a Gemini varian $200 \mathrm{MHz}$.

\section{Typical procedures}

A mixture of furan $(30 \mathrm{mmol}, 3.00 \mathrm{~g})$, methyl bromide $(10 \mathrm{mmol}, 0.81 \mathrm{~g})$ and TOAB $(0.3 \mathrm{~g})$ was taken in a $100 \mathrm{~mL}$ round bottom Pyrex flask. The reaction was carried and supported under $\mathrm{N}_{2}$. Once the reaction completed (Monitored by GC), the reaction mass was filtered, distilled off to remove $\mathrm{HBr}$ and unreacted reagents and substrates and then purified.

Spectral data

${ }^{1} \mathrm{H}$ NMR spectrum of 2-methy thiophene (Figure 1):

$\delta=2.43(3 \mathrm{H}, \mathrm{s}), \delta=7.9(1 \mathrm{H}, \mathrm{dd}), \delta=7.4(1 \mathrm{H}, \mathrm{d}), \delta=7.18(1 \mathrm{H}, \mathrm{d}) .{ }^{13} \mathrm{C}$ NMR $\left(\mathrm{dmso}^{-} \mathrm{d}_{6}, 100\right.$ $\mathrm{MHz}$ ) 138.7, 127.8, 127.0, 125.10,15.12.HRMS (ESI) calcd for $\left[\mathrm{C}_{5} \mathrm{H}_{7} \mathrm{~S}\right]{ }^{+}$99.17. Found: 99.16. FT-IR $\left(\mathrm{v} \mathrm{cm}^{-1}\right) 29852$. 


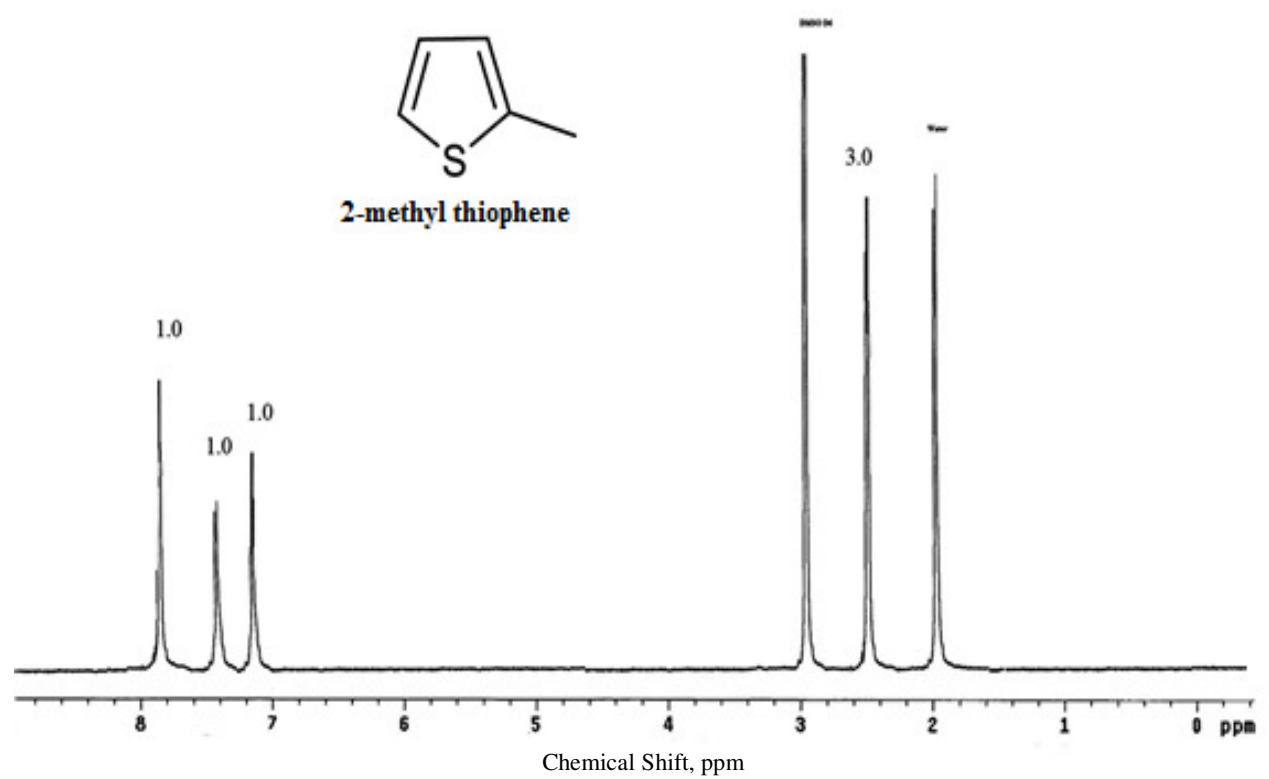

Figure 1. ${ }^{1} \mathrm{H}$ NMR spectrum of 2-methy thiophene

${ }^{1} \mathrm{H}$ NMR spectrum of 2-methyl furan (Figure 2):

$\delta=2.23(3 \mathrm{H}, \mathrm{s}), \delta=7.00(1 \mathrm{H}, \mathrm{m}) .{ }^{13} \mathrm{C}$ NMR $\left(\right.$ dmso-d $\left._{6}, 100 \mathrm{MHz}\right) 152.10,142.4,110.11$, 105.20, 15.00. HRMS (ESI) calcd for $\left[\mathrm{C}_{5} \mathrm{H}_{7} \mathrm{O}\right]^{+} 83.14$. Found: 83.11.

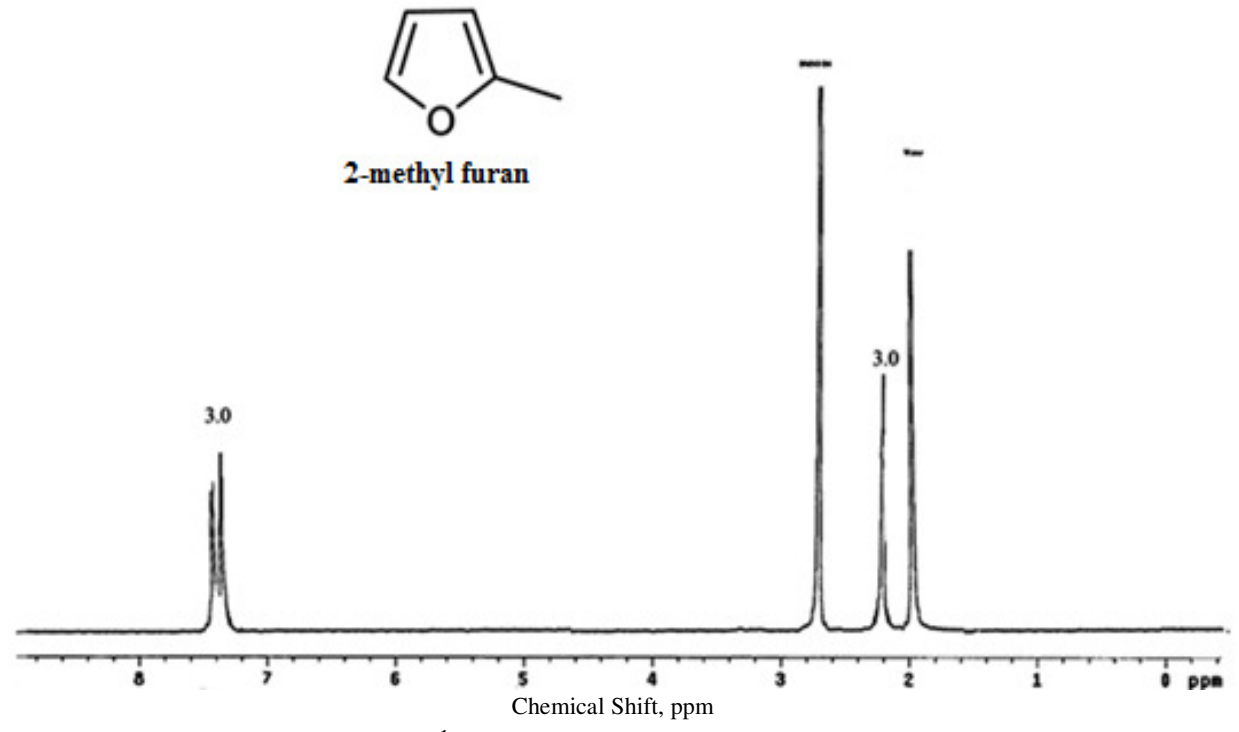

Figure 2. ${ }^{1} \mathrm{H}$ NMR spectrum of 2-methyl furan

${ }^{1} \mathrm{H}$ NMR spectrum of 2-methyl pyrrole (Figure 3):

$\delta=2.30(3 \mathrm{H}, \mathrm{s}), \delta=8.38(1 \mathrm{H}, \mathrm{t}), \delta 8.12(1 \mathrm{H}, \mathrm{m}), \delta=7.91(1 \mathrm{H}, \mathrm{d}), \delta=10.0(1 \mathrm{H}, \mathrm{brs}) .{ }^{13} \mathrm{C}$ NMR (dmso-d $\left.\mathrm{d}_{6} 100 \mathrm{MHz}\right) 128.4,118.0,108.3,107.2,17.0 . \mathrm{HRMS}$ (ESI) calcd for $\left[\mathrm{C}_{5} \mathrm{H}_{8} \mathrm{~N}\right]$ ${ }^{+} 82.30$ Found: 82.13 . 


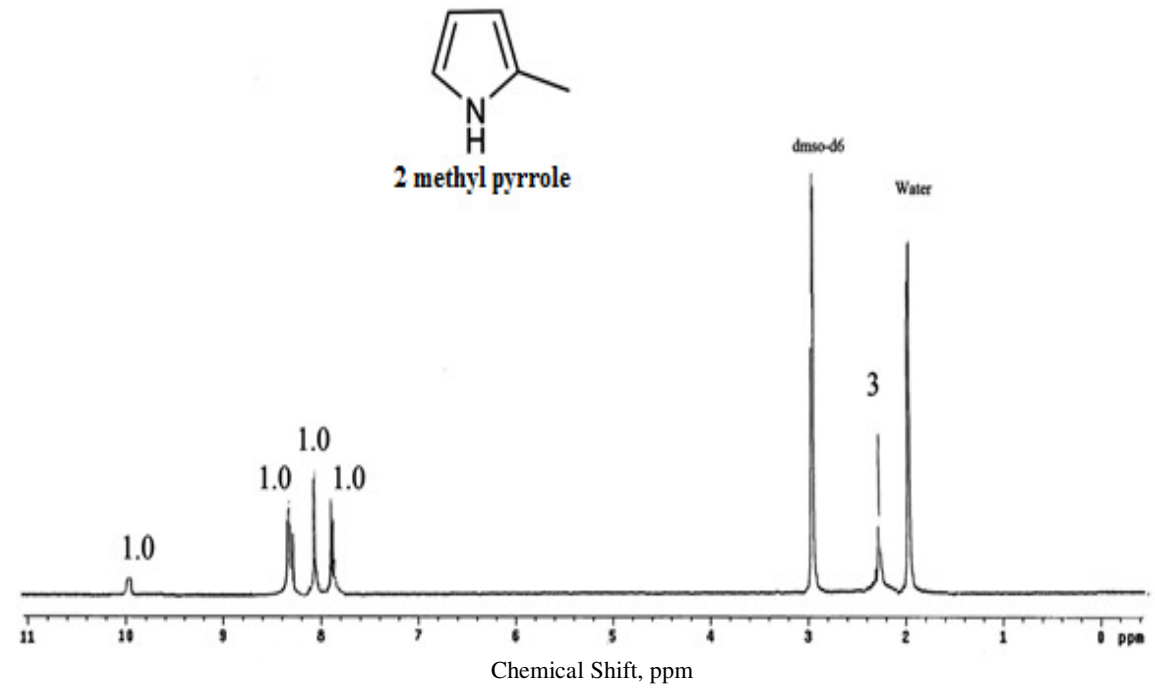

Figure 3. ${ }^{1} \mathrm{H}$ NMR spectrum of 2-methyl pyrrole

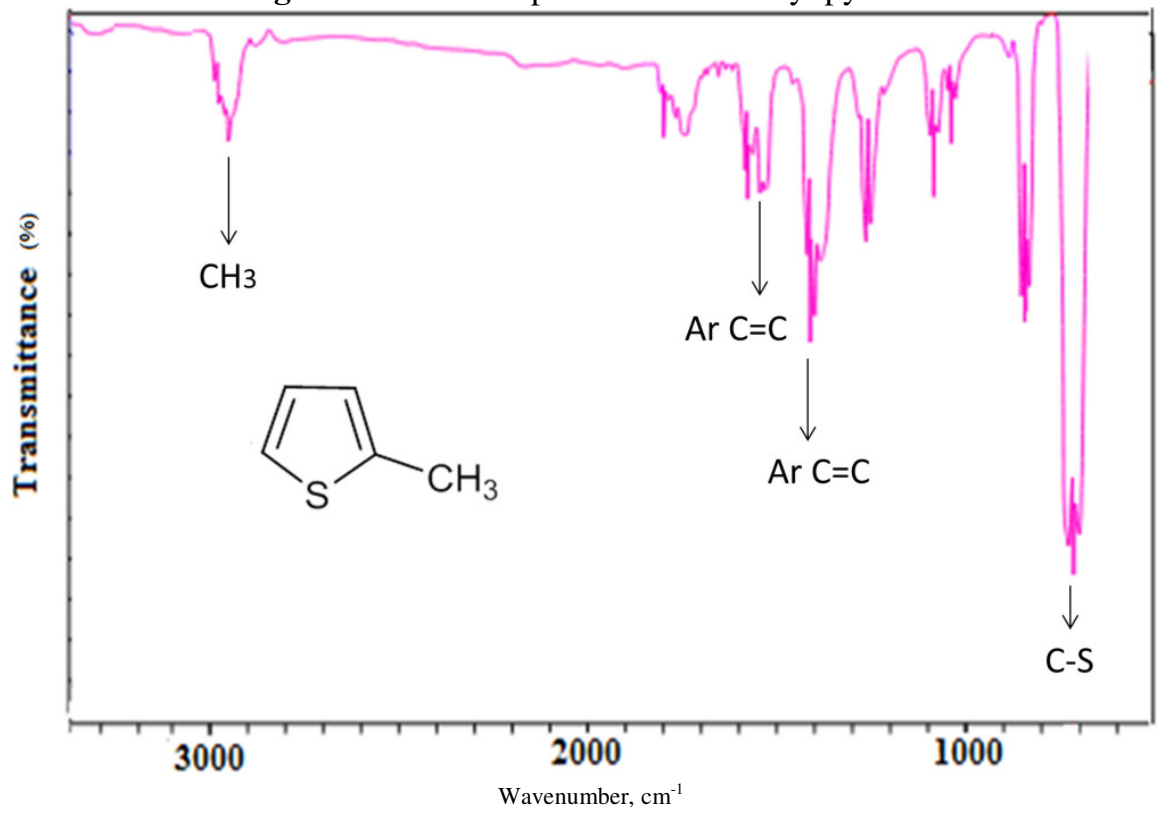

Figure 4. FT-IR spectra of 2-methyl pyrrole

\section{Results and Discussion}

The phase transfer catalysed reaction i.e alkylation of 5 membered aromatic heterocycles is shown in the Scheme 1. The results about this catalyst action or choosiness of various temperature conditions in the alkylation procedure of these significant organic intermediates thiophene, pyrrole and furan with methyl bromide directed are embodied in Table 1. The carbon alkylation (C-Alkylation) of furan was carried out using TOAB has displayed higher activity $^{7-9}$. Using this well-organized catalyst, thiophene and pyrrole are also alkylated. 
The selectivity and conversion of 2-alkyalted heterocyclics are optimized with various temperatures such as RT, 35, 40 and $100{ }^{\circ} \mathrm{C}$. In the methylation process of furan, pyrrole and thiophene at $100{ }^{\circ} \mathrm{C}$ the conversion was increased with the enhanced selectivity. Some 3alkylated product was found to be the predominant minor product. The reactions enthused at ambient temperature to give 2-alkylated yields in practically pure form. The higher discernment is qualified to the kinetic effect. Selective alkylation of furan to 2-methyl furan is self-governing of temperatures used in methylation, conducted in presence of phase transfer catalyst TOAB, the formation of 3-alkyl product is not noticed in selected reaction condition either. So, the highly desired and pure isomers are appreciated using low quantity or very ignored quantity of PTC. The aromatic methyl protons are showed their significant proton NMR spectral signals for compounds 2-methy thiophene (Figure 1), 2-methyl furan (Figure 2), 2-methyl pyrrole (Figure 3) at $2.43 \mathrm{ppm}, 2.23 \mathrm{ppm}$ and $2.30 \mathrm{ppm}$ respectively. The peculiar compound 2-methyl pyrrole (Figure 3) had been showed its $\mathrm{NH}$ signal at $10.00 \mathrm{ppm}$ due to the ring strain and electronegativity of the Nitrogen ${ }^{11-13}$ (3 most electronegative element after Fluorine and Oxygen). Further in the FT-IR (Figure 4, Compound 2-methy thiophene) spectra of the methyl group strech was observed at $2880 \mathrm{~cm}^{-1}$ which is also proved the successful methylation in the reaction.

\section{Catalytic activity}

In the instance of improvement of the alkylation (Scheme 1), we have discovered various TOAB as the best catalyst at temperature $100{ }^{\circ} \mathrm{C}$ rather than temperature range RT-100 ${ }^{\circ} \mathrm{C}$ (Table 1). PTC supported reactions for any alkylation with at different can give moderate to good yields of desired products. This because of the presence of higher number of carbon atoms in the TOAB releases the quartenary ammonium cation, in its equilibrium mechanism which in-turn supports the nucleophilic substitution of the methyl cation onto the heterocyclcic ring. TOAB display both Bronsted and Lewis acid sites for the alkylation of our substrates i.e., quarternary ammonium cation and bromide ion. The strong electronegative element $\left(\mathrm{Br}^{-}\right)$actively attacks at the second position of the heterocyclic ring to release the $\mathrm{HBr}$ by creating the resonance hybrids of thiophene, furan, and pyrrole. These newly formed negatively resonance hybrids readily participate in ionic bond formation between quarternary ammonium cation $\left(\mathrm{Q}^{+}\right)$and themselves. This salt was then approached by $\mathrm{CH}_{3}{ }^{+}$to form stabilized aromatic ring by 2-methyl heterocyclic ring by evolving the $\mathrm{HBr}$ and TOAB. The alkylation of the heteroaromatic molecules are highly selective at aposition $^{7-10}$ of the ring with the selected catalyst and to yield the higher product.

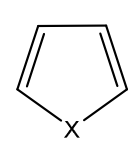

\section{Conclusion}

The phase transfer catalyst TOAB offers the highly effective and selective alkylation process for the desired organic intermediates, by using alkyl halide at $100{ }^{\circ} \mathrm{C}$ under inert atmosphere $\mathrm{N}_{2}$. Though this method was well established, here we report the accuracy of the temperature which could be more useful. This catalyst was reported here which never produce any sewages unlike Lewis acid and their corresponding acids. Our approach described here is not only ecological but also an inexpensive 


\section{Acknowledgement}

Authors are thankful to adiyta group of companies, Hyderabad for providing all required chemicals, and special thanks to MB.

\section{References}

1. Susanne R, Alexander S, Jens P, Thomas L, Josef P, Karl-Heinz S, Peter R and Elisabeth P, J Med Chem., 2005, 48(19), 5900-5908; DOI:10.1021/jm050101d

2. Neil F G and Grant M W, J Chem Engg Data, 2006, 51(6), 1973-1981; DOI:10.1021/je050473r

3. Varun B,Divya G, Vikrant A, Saurabh D and Poonam S, RSC Adv., 2015, 5, 15233 15236, DOI: $10.1039 / \mathrm{c} 4 \mathrm{ra} 15710 \mathrm{a}$

4. DeCastro C, Sauvage E, Valkenberg M H and Holderich W F, J Cataly., 2000, 196(1), 86-94; DOI:10.1006/jcat.2000.3004

5. George A. O, Joseph K and Josef B, J Org Chem., 1977, 42(26), 4187-4191; DOI:10.1021/jo00862a003

6. Yoshiaki N, Yuuya Y, Natsuko K and Tamejiro H, J Am Chem Soc., 2010, 132(39), 13666-13668; DOI:10.1021/ja106514b

7. Yoshinori Y, Shinichi Y, Hidetaka Y and Kazuhiro M, J Am Chem Soc., 1980, 102(7), 2318-2325; DOI:10.1021/ja00527a032

8. Maw-Ling W, Manuri B and Hsieh Y-M, J Taiwan Institute Chem Engs., 2015, 57, 5461; DOI:10.1016/j.jtice.2015.05.039

9. Manuri B and Maw-Ling W, J Taiwan Institute Chem Engs., 2014, 45(6), 2899-2905; DOI:10.1016/j.jtice.2014.08.010

10. Manuri B, Shenghong A D and Shing-Yi S, RSC Advances, 2015, 5, 65351-65357; DOI:10.1039/C5RA08910G

11. Viplava Prasad U, Brahmayya M, Samsonu D, Vijayakumar K, Raghupathiraju M V, Sci Rev Chem Commun., 2011, 1(1), 58-62.

12. Raghav M, Jha K K, Sachin K and Isha T, Der Pharma Chemica, 2011, 3, 38-54.

13 Brahmayya M and Wang M L, Res J Pharma Biolog Chem Sci., 2014, 5(3), 565-571.

14. Brahmayya M, Wang M L, Res J Pharmal Biolog Chem Sci., 2014, 5(3), 751-755. 